\title{
The Prevalence and Characterization of Extended-Spectrum $\beta$-Lactamase- and Carbapenemase-Producing Bacteria from Hospital Sewage, Treated Effluents and Receiving Rivers
}

\author{
Luhua Zhang ${ }^{1}{ }^{(0,}$, Xinyue $\mathrm{Ma}^{2}, \mathrm{Li} \mathrm{Luo}^{2}$, Nan Hu ${ }^{1}$, Jiayao Duan ${ }^{1}$, Zhongjian Tang ${ }^{1}$, \\ Rujie Zhong ${ }^{1}$ and Ying $\mathrm{Li}^{2, *}$ \\ 1 Department of Pathogenic Biology, School of Basic Medical Sciences, Southwest Medical University, \\ Luzhou 646000, China; zhluhua@swmu.edu.cn (L.Z.); h20200210@126.com (N.H.); d991806@163.com (J.D.); \\ tt403921424@163.com (Z.T.); Evenlee1987@163.com (R.Z.) \\ 2 Department of Immunology, School of Basic Medical Sciences, Southwest Medical University, \\ Luzhou 646000, China; HMM151126@126.com (X.M.); chenxishiwei@126.com (L.L.) \\ * Correspondence: Lying1019@swmu.edu.cn; Tel.: +86-0830-3160-073
}

Received: 13 January 2020; Accepted: 10 February 2020; Published: 13 February 2020

\begin{abstract}
Hospital sewage plays a key role in the dissemination of antibiotic-resistant genes (ARGs) by serving as an environmental antimicrobial resistance reservoir. In this study, we aimed to characterize the cephalosporin- and carbapenem-resistant isolates from hospital sewage and receiving rivers. The results showed that ESBL ( $\left.b l a_{\mathrm{CTX}-\mathrm{M}}\right)$ and carbapenemase genes (bla $a_{\mathrm{NDM}}$ and $\left.b l a_{\mathrm{KPC}}\right)$ were widely detected in a number of different bacterial species. These resistance genes were mainly harbored in Enterobacteriaceae, followed by Acinetobacter and Aeromonas isolates. More attention should be given to these bacteria as important vectors of ARGs in the environment. Furthermore, we showed that the multidrug resistance phenotype was highly prevalent, which was found in $85.5 \%$ Enterobacteriaceae and $75 \%$ Acinetobacter strains. Notably, the presence of carbapenemase genes in isolates from treated effluents and receiving rivers indicates that the discharges of wastewater treatment plants could be an important source for high-risk resistance genes propagation to the environment. In conclusion, this study shows a high prevalence of ESBL- and carbapenemase-producing bacteria in hospital sewage and receiving rivers in China. These findings have serious implications for human health, and also suggest the need for more efforts to control the dissemination of resistant bacteria from hospital sewage into the environment.
\end{abstract}

Keywords: carbapenemase; $b l a_{\mathrm{NDM}}$; $b l a_{\mathrm{KPC}}$; hospital sewage; Enterobacteriaceae

\section{Introduction}

The emergence and rapid dissemination of antibiotic resistance is a serious and growing problem for human health [1]. $\beta$-lactam antibiotics are by far the most used antibiotics worldwide for treating infections in both humans and animals [2]. A significant threat to the usage of these agents is the rapid evolution of $\beta$-lactamases, mainly among Gram-negative bacteria, which makes each new drug obsolete in a very short period of time [3]. Among the $\beta$-lactams, carbapenem antibiotics are considered to be the most reliable last-resort treatment for bacterial infections caused by extended-spectrum $\beta$-lactamases (ESBLs)-producing bacteria [4]. The rapid spread of carbapenem resistance, usually caused by the production of carbapenemase, constitutes a critical public-healthcare problem worldwide [4]. Carbapenemases have the ability to hydrolyze penicillins, cephalosporins and carbapenems, thereby limiting treatment options [5]. Studies have shown an increased mortality associated with serious 
carbapenem-resistant Enterobacteriaceae (CRE) infections among hospitalized adults [6,7]. Various types of carbapenemases have been reported, among which the most commonly reported enzymes are the Ambler class A $\beta$-lactamases (e.g., KPC), the class B $\beta$-lactamases/metallo- $\beta$-lactamases (e.g., NDM and IMP) and the class D oxacillinases (e.g., OXA-48 and OXA-58) [8]. These carbapenemase genes are frequently located on mobile genetic elements and can be transferred among bacteria from different genera [9]. Particularly, bacteria belonging to the family Enterobacteriaceae and Acinetobacter usually function as important vectors in the dissemination of $\beta$-lactamase genes in natural bacterial ecosystems [10,11].

There is an increasing concern regarding the growing spread of antibiotic-resistant bacteria (ARB) in the environment. Sewage discharged from hospitals is a complex matrix with a collection of feces and urine of patients undergoing intensive antibiotic treatment and wastewater containing ARB from clinical settings. It serves as a reservoir of antibiotic resistance and a hotspot for horizontal gene transfer, enabling the spread of antibiotic resistance genes (ARGs) between bacterial communities [12]. Previous studies have identified a high abundance of ARB in hospital sewage around the world [13-18]. The first report concerning carbapenemases-producing bacteria (CPB) in hospital sewage in China

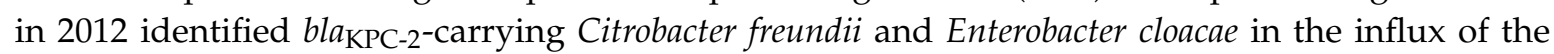
wastewater treatment plants (WWTPs) [19]. After that, studies have detected a rich variety of resistant bacteria that produce carbapenemases, including NDM-1, KPC-2, and OXA-58 in hospital sewage in China [20-23]. Rivers are natural receptacles for large amounts of microbial contaminants from domestic sewage, livestock wastewater, and hospital effluents. Previous studies have also reported the isolation of CPB from water samples of rivers in different countries, such as KPC-2-producing C. freundii in China [24], OXA-48-like-producing Escherichia coli and Klebsiella pneumoniae in Algeria [25], and NDM-9-producing Klebsiella variicola in South Korea [26]. Studies by Azuma et al. and Khan et al. highlighted the dissemination of clinically significant ARB from hospitals into the receiving water bodies $[27,28]$. However, very limited studies have been conducted concerning the diversity and prevalence of ARGs and ARB in hospital sewage systems and receiving waters in China.

The aim of this study was, therefore, to (i) investigate the distribution and prevalence of carbapenemase- and ESBL-encoding genes from hospital sewage systems and receiving rivers in Southwest China, and (ii) characterize the pollution levels, with a focus on the Enterobacteriaceae and Acinetobacter isolates. Understanding the presence and spreading pathway of carbapenemase and ESBL genes in the hospital sewage and receiving waters are critical to assess their dissemination risk to public health through the aquatic environment.

\section{Materials and Methods}

\subsection{Sample Collection}

In August 2019, a total of six samples of hospital raw sewage and treated effluents were collected from hospital WWTPs of three tertiary care hospitals, with hospital A (2200 beds) being located in the center, hospital B (3000 beds) in the north, and hospital C (1000 beds) in the east of Luzhou City in Sichuan province, China. Five surface water samples were collected along receiving rivers influenced by these three hospitals. Each sampling site is at least $1 \mathrm{~km}$ apart (Figure 1). Water samples $(500 \mathrm{~mL})$ were collected during weekdays in the morning between $9.30 \mathrm{a} . \mathrm{m}$. and $11 \mathrm{a} . \mathrm{m}$. with sterile plastic bottles and taken to the laboratory for subsequent analysis within the following $1 \mathrm{~h}$. 


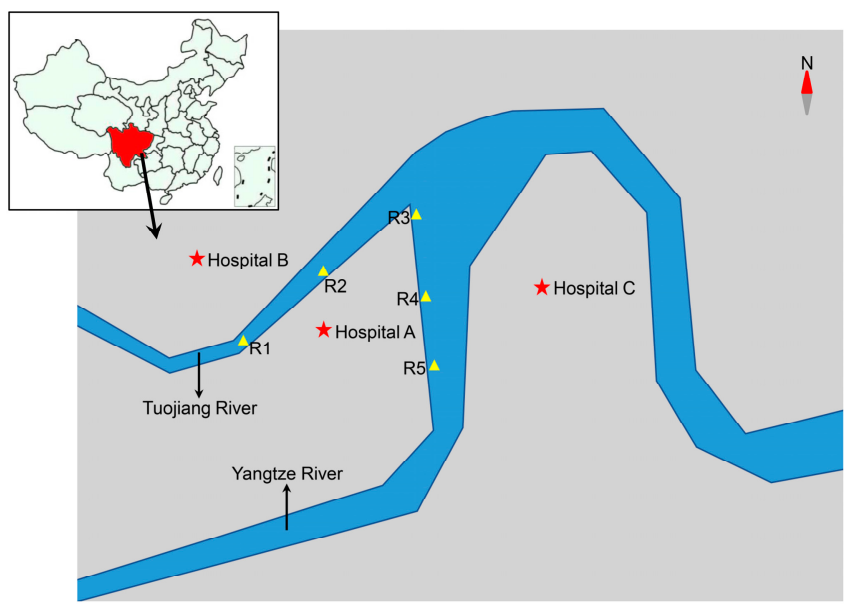

Figure 1. Schematic diagram of sampling sites of hospital sewage and river water, Luzhou City, Sichuan, China, 2019. The sampling sites of hospital sewage are indicated by stars. R1-R5: sampling sites of river water.

\subsection{Isolation of ESBL- and Carbapenem-Resistant Gram-Negative Bacteria}

Bacterial cells in water samples $(6 \mathrm{~mL}$ for raw sewage, $120 \mathrm{~mL}$ for effluent samples and $200 \mathrm{~mL}$ for river water) were collected by centrifugation at $5000 \times \mathrm{g}$ for $5 \mathrm{~min}$, and resuspended in sterile $0.9 \% \mathrm{NaCl}$ solution. Ten-fold serial dilutions (10-1000 times) of each water sample were made in sterile saline solution. A total of $100 \mu \mathrm{L}$ of each dilution was then plated onto MacConkey agar supplemented with cefotaxime $(4 \mu \mathrm{g} / \mathrm{mL})$ or meropenem $(2 \mu \mathrm{g} / \mathrm{mL})$ for rapid screening of potential ESBL- or carbapenemase-producing Gram-negative bacteria. After incubated for $24 \mathrm{~h}$ at $37^{\circ} \mathrm{C}$, bacterial colonies with distinct coloration and morphologies were randomly picked and subcultured onto MacConkey agar containing cefotaxime $(4 \mu \mathrm{g} / \mathrm{mL})$ or meropenem $(2 \mu \mathrm{g} / \mathrm{mL})$ for further purification. A single colony of purified bacteria was picked and cultured in Trypticase Soy Broth. Each isolate was assigned a unique identification number and stored at $-80{ }^{\circ} \mathrm{C}$ in $25 \%$ (vol/vol) glycerol for further investigation.

\subsection{Bacterial Species Identification}

Rapid bacterial genomic DNA extraction of selected isolates was performed by boiling method. Species identification was performed by PCR amplifying of the 16S rRNA gene using the primer pair 27F/1492R [29]. The PCR products were purified and then sequenced using Sanger sequencing by Tsingke Biotech (Beijing, China). Resulting nucleotide sequences of 16S rRNA genes were compared with sequences in GenBank (NCBI) database using BLAST software.

\subsection{Detection of Antibiotic Resistance Genes}

All the isolates were examined for the presence of ten $\beta$-lactamase genes, including $b l a_{\mathrm{CTX}-\mathrm{M}}$, $b l a_{\mathrm{KPC}}, b l a_{\mathrm{NDM}}, b l a_{\mathrm{OXA}-48}, b l a_{\mathrm{OXA}-58}, b l a_{\mathrm{IMP}}, b l a_{\mathrm{VIM}}$ by PCR using specific primers (Table S1). All the PCR products were analyzed on $1.5 \%$ agarose gel by electrophoresis. Gene identification was further performed on all isolates positive for $b l a_{\mathrm{CTX}-\mathrm{M}}$ using primers $b l a_{\mathrm{CTX}-\mathrm{M}-1 \text { group }}, b l a_{\mathrm{CTX}-\mathrm{M}-2 \text { group, }}$ $b l a_{\mathrm{CTX}-\mathrm{M}-8 \text { group }}$, and $b l a_{\mathrm{CTX}-\mathrm{M}-9 \text { group. Sequences of } b l a_{\mathrm{CTX}-\mathrm{M}-1 / 2 / 8 / 9} \text { group }}, b l a_{\mathrm{KPC}}$, and $b l a_{\mathrm{NDM}}$ amplicons were determined using Sanger sequencing by Tsingke Biotech, and were compared with reported sequences available in the NCBI database using BLAST software.

\subsection{Antimicrobial Susceptibility Testing}

All Enterobacteriaceae isolates were evaluated for resistance to different antibiotic agents by the disk diffusion method according to the guidance of Clinical and Laboratory Standards Institute (CLSI) M100 [30]. The antibiotics tested were amoxicillin-clavulanic acid (AMC, $30 \mu \mathrm{g}$ ), ampicillin (AMP, 
$10 \mu \mathrm{g})$, ciprofloxacin (CIP, $5 \mu \mathrm{g})$, tetracycline (TET, $30 \mu \mathrm{g})$, gentamicin $(\mathrm{CN}, 10 \mu \mathrm{g})$, chloramphenicol $(\mathrm{C}, 30 \mu \mathrm{g})$, streptomycin $(\mathrm{S}, 10 \mu \mathrm{g})$, cefoxitin (FOX, $30 \mu \mathrm{g})$, cefotaxime (CTX, $30 \mu \mathrm{g})$, meropenem (MEM, $10 \mu \mathrm{g}$ ), and trimethoprim/sulfamethoxazole (SXT, $25 \mu \mathrm{g}$ ). In addition, all the Acinetobacter isolates were tested for susceptibility to CIP, TET, CN, FOX, CTX, MEM, and SXT using the disk diffusion method according to the CLSI. All the tested isolates were categorized as "S (sensitive)" or "R (resistant)" (Intermediate resistance, with inhibition zones larger than $\mathrm{R}$ and smaller than S, was excluded from the resistance percentage calculations). Mueller-Hinton agar (Solarbio, Beijing, China) was used for all assays, and zone diameters were determined following incubation at $37^{\circ} \mathrm{C}$ for 16 to $24 \mathrm{~h}$. Interpretation of zone diameters was done according to the CLSI criteria. E. coli ATCC 25,922 and Pseudomonas aeruginosa ATCC 27,853 were used as control in this study. The isolate was considered as multidrug-resistant (MDR), when it was resistant to three or more classes of antimicrobial agents.

\section{Results}

\subsection{Bacterial Isolation}

In the present study, a total of 104 non-repetitive Gram-negative bacteria were picked from MacConkey agar containing cefotaxime $(n=54,51.9 \%)$ or meropenem $(n=50,48.1 \%)$. Of them, $66(63.5 \%)$ isolates were collected from raw sewage (hospital $A, n=20$; hospital $B, n=20$; hospital C, $n$ $=26), 17(16.3 \%)$ from effluent samples (hospital $\mathrm{A}, \mathrm{n}=3$; hospital $\mathrm{B}, \mathrm{n}=2$; hospital $\mathrm{C}, \mathrm{n}=12$ ), and 21 $(20.2 \%)$ were isolated from river water (Figure 1$)$.

\subsection{Bacterial Identification}

A total of 62 (59.6\%) out of the 104 isolates were found to be Enterobacteriaceae; 35/62 (56.5\%) were E. coli, 17/62 (27.4\%) K. pneumoniae, 5/62 (8.1\%) Enterobacter spp., 3/62 (4.8\%) Citrobacter spp., 2/62 (3.2\%) Raoultella planticola, and 2/63 (3.2\%) K. variicola. The remaining isolates were identified as: Acinetobacter spp. (20/104, 18.3\%), Aeromonas spp. (11/104, 10.6\%), Pseudomonas spp. (6/104, 5.8\%), Comamonas spp. $(2 / 104,1.9 \%)$, Stenotrophomonas maltophilia $(1 / 104,0.96 \%)$, Cupriavidus taiwanensis $(1 / 104,0.96 \%)$ and Proteus mirabilis (1/104, 0.96\%).

Of the 66 isolates from raw sewage, the most predominant taxa were E. coli $(25 / 66,37.9 \%)$, K. pneumoniae (11/66, 16.7\%), and Acinetobacter spp. (11/66, 16.7\%). Resistant strains identified in effluent samples were dominated by Pseudomonas spp. (5/17, 29.4\%), Acinetobacter spp. (5/17, 29.4\%), and E. coli $(4 / 17,23.5 \%)$. The most commonly identified isolates from river water were E. coli $(6 / 21$, 28.6\%), followed by K. pneumoniae (5/21, 23.8\%) and Acinetobacter spp. (4/21, 19\%).

\subsection{Detection and Molecular Characterization of Resistance Genes}

Of the 104 isolates, bla $a_{\mathrm{CTX}-\mathrm{M}}$-type gene was identified in a total of 69/104 (66.3\%) strains. CTX-Ms were mainly harbored in species of Enterobacteriaceae $(52 / 69,75.4 \%)$, including E. coli (31/53), K. pneumoniae (11/53), Enterobacter spp. (4/53), K. variicola (2/53), Citrobacter spp. (1/53), and R. planticola $(1 / 53)$ (Figure 2a). At least ten different CTX-M variants were identified in CTX-M producers, including CTX-M-55 ( $\mathrm{n}=31)$, CTX-M-3 ( $\mathrm{n}=7)$, CTX-M-14 ( $=$ 6), CTX-M-15 $(\mathrm{n}=5)$, CTX-M-65 ( $\mathrm{n}=4)$, CTX-M-27 $(\mathrm{n}=3)$, CTX-M-199 $(\mathrm{n}=2)$, CTX-M-123 ( $\mathrm{n}=2)$, CTX-M-9 $(\mathrm{n}=1)$, and CTX-M-213 $(\mathrm{n}=1)$. The subtypes of $b l a_{\mathrm{CTX}-\mathrm{M}}$ in seven isolates were not determined in this work.

The most frequently detected carbapenemase-encoding gene in this work was $b l a_{\mathrm{NDM}}$ and it was present in 38/104 (36.5\%) isolates (Figure $2 b$ ), of which 25 were recovered from raw sewage, 8 from effluent samples, and 5 from river water. Bla $a_{\mathrm{NDM}}$ was frequently detected in species of Enterobacteriaceae, including E. coli $(\mathrm{n}=13)$, K. pneumoniae $(\mathrm{n}=4)$, and Citrobacter spp. $(\mathrm{n}=2)$. It was noteworthy that a high detection rate of bla $a_{\mathrm{NDM}}$ was also observed in Acinetobacter spp. (13/20,65\%). Sequencing analysis confirmed that the most frequently identified $b l a_{\mathrm{NDM}}$ variant was $b l a_{\mathrm{NDM}-1}(19 / 38,50 \%)$, followed by $b l a_{\mathrm{NDM}-5}(18 / 38,47.4 \%)$. A $b l a_{\mathrm{NDM}-24}$ gene was found to be present in one Comamonas sp. isolate from raw sewage. 


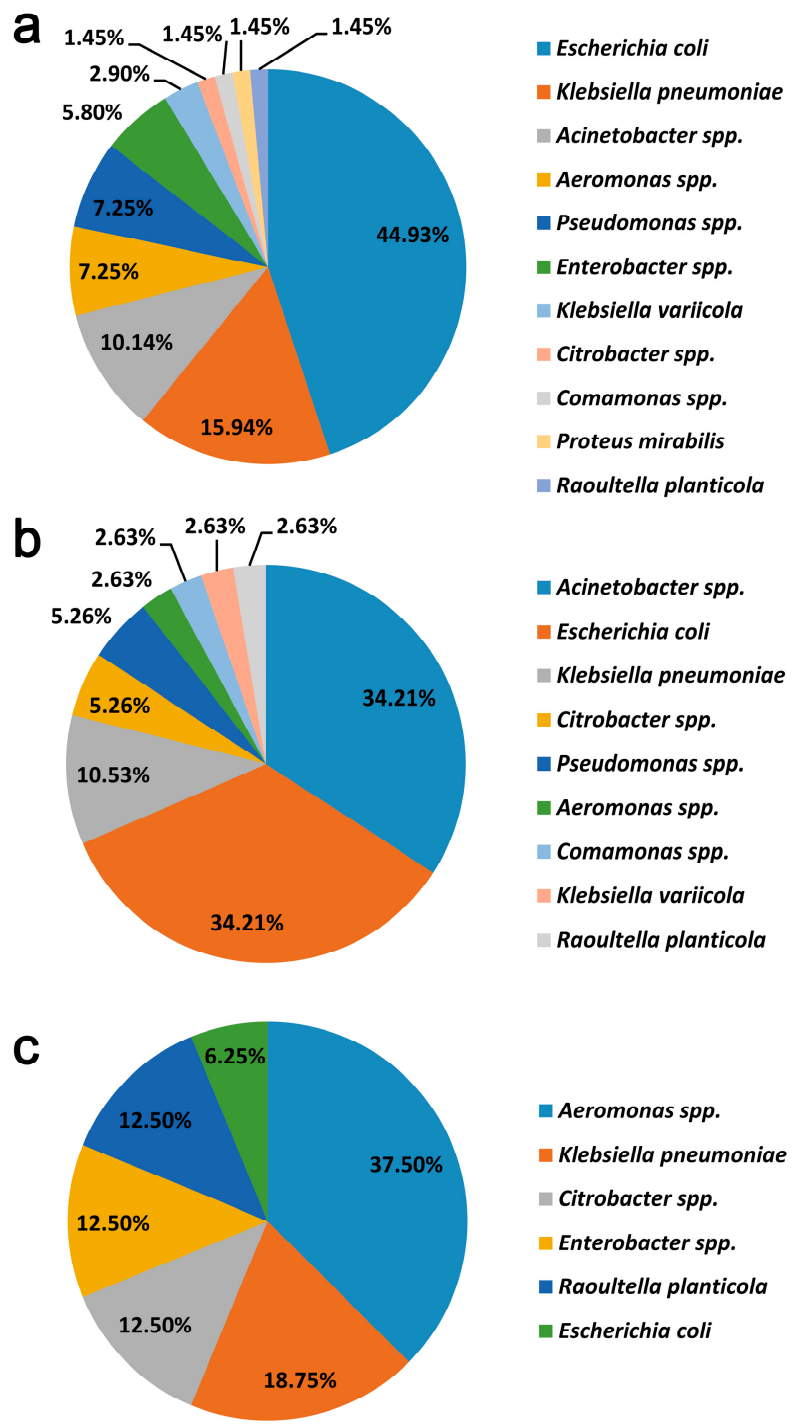

Figure 2. Distribution of the $b l a_{\mathrm{CTX}-\mathrm{M}}(\mathbf{a}), b l a_{\mathrm{NDM}}(\mathbf{b})$, or $b l a_{\mathrm{KPC}}(\mathbf{c})$ in the collected isolates in this study.

The second most prevalent carbapenemase-encoding gene was $b l a_{\mathrm{KPC}-2}$, which was identified in $16 / 104(15.4 \%)$ isolates (Figure 2c). A high proportion $(n=15,93.8 \%)$ was isolated from raw sewage and that which remained was from an effluent sample. Bla $a_{\mathrm{KPC}-2}$ was mostly identified in Enterobacteriaceae, including three K. pneumoniae, two Enterobacter spp., two Citrobacter spp., 2 R. planticola, and one E. coli. It was notable that $b l a_{\mathrm{KPC}-2}$ was also detected in six Aeromonas strains. Additionally, $b l a_{\mathrm{OXA}-58}$ was detected in seven $(7 / 104,6.7 \%)$ Acinetobacter isolates (five from raw sewage and two from effluent samples). One Aeromonas isolate $(1 / 104,0.96 \%)$ from raw sewage was found to harbor $b a_{\text {IMP }}$ gene, which was further identified to be $b l a_{\mathrm{IMP}-4}$ by whole genome sequence analysis of this strain (data not shown). None of the isolates were positive for OXA48-type or VIM-type carbapenemases.

In addition, 28 out of 104 resistant strains $(26.9 \%)$ coproduced CTX-M and carbapenemases, harbored by isolates of Enterobacteriaceae $(\mathrm{n}=19)$, Acinetobacter spp. $(\mathrm{n}=4)$, Aeromonas spp. $(\mathrm{n}=3)$ and Pseudomonas spp. $(\mathrm{n}=2)$. Of these resistant strains, $18(64.3 \%)$ were recovered from raw sewage, nine $(32.1 \%)$ from effluent samples and one $(3.6 \%)$ from river water. The coexistence of NDM and OXA-58 was identified in seven Acinetobacter strains (five from raw sewage and two from effluent samples). Carbapenemase genes $b l a_{\mathrm{KPC}-2}$ and $b l a_{\mathrm{IMP}-4}$ were coharbored in an Aeromonas isolate from raw sewage. Additionally, two Enterobacteriaceae strains, one Citrobacter sp. and one R. planticola from raw sewage, were found to carry both $b l a_{\mathrm{NDM}-1}$ and $b l a_{\mathrm{KPC}-2}$ genes in addition to $b l a_{\mathrm{CTX}-\mathrm{M}-14}$. 


\subsection{Antimicrobial Susceptibility of Enterobacteriaceae and Acinetobacter Isolates}

Analysis of the antimicrobial resistance of 62 Enterobacteriaceae strains (Table 1) revealed that all of them were resistant to ampicillin and cefotaxime. Furthermore, they presented a relatively high resistance to trimethoprim/sulfamethoxazole (77.4\%) and tetracycline $(74.2 \%)$, followed by amoxicillin-clavulanic acid (66.1\%), cefoxitin (61.3\%), and ciprofloxacin (61.3\%). Medium resistance levels were found to gentamicin (51.6\%), chloramphenicol (48.4\%), and streptomycin (41.9\%). A total of 32/62 (51.6\%) Enterobacteriaceae strains were identified to be meropenem-resistant, of which 29 were positive for carbapenemase-encoding genes. Multidrug-resistant (MDR) strains, which were defined as resistance to at least 3 classes of antimicrobial agents, were commonly observed in the tested Enterobacteriaceae isolates by $85.5 \%$ (53/62). A total of $91.4 \%(32 / 35)$ E. coli and $94.1 \%(16 / 17)$ K. pneumoniae isolates were identified to be MDR. CRE strains isolated in this study showed a high percentage $(28 / 32,87.5 \%)$ of multi-drug resistance. It was noted that nine isolates demonstrated resistance to all 11 antimicrobial agents, including six NDM-5-producing E. coli (five from raw sewage and one from river water), two NDM-5-producing K. pneumoniae (one from effluent samples and the other from river water), and one NDM-5-producing $C$. freundii from river water.

Antibiotic susceptibility studies of Acinetobacter isolates revealed high levels of resistance to clinically important antibiotics (Table 2$)$, including gentamicin $(55 \%)$, cefoxitin $(85 \%)$, cefotaxime $(100 \%)$, tetracycline (75\%), and trimethoprim/sulfamethoxazole (65\%). However, Acinetobacter isolates showed a low resistance to ciprofloxacin (10\%). It was worth noting that 14/20 (70\%) Acinetobacter isolates showed resistance to meropenem, among which, 13 were found to carry at least one carbapenemase-encoding gene. Meanwhile, 75\% (15/20) of Acinetobacter strains were found to be resistant to three or more antibiotic classes tested. 
Table 1. Enterobacteriaceae isolated from aquatic environments.

\begin{tabular}{|c|c|c|c|c|c|c|c|c|c|c|c|c|c|c|c|c|}
\hline Isolation No. $^{a}$ & Species & Isolation Source & $\mathrm{AMP}^{\mathrm{b}}$ & CTX & FOX & MEM & AMC & $\mathrm{CN}$ & $\mathrm{S}$ & $\mathrm{C}$ & TET & CIP & SXT & NDM & KPC & CTX-M \\
\hline FA8C & Klebsiella pneumoniae & hospital A/raw sewage & $\mathrm{R}$ & $\mathrm{R}$ & S & S & $\mathrm{R}$ & $\mathrm{R}$ & $\mathrm{R}$ & $\mathrm{R}$ & $\mathrm{R}$ & $\mathrm{R}$ & $\mathrm{R}$ & & & 3 \\
\hline FA9C & Escherichia coli & hospital A/raw sewage & $\mathrm{R}$ & $\mathrm{R}$ & $\mathrm{s}$ & $\mathrm{S}$ & $\mathrm{S}$ & S & $\mathrm{S}$ & $\mathrm{S}$ & S & $\mathrm{R}$ & S & & & 3 \\
\hline FA11C & Klebsiella pneumoniae & hospital A/raw sewage & $\mathrm{R}$ & $\mathrm{R}$ & S & S & I & I & S & $\mathrm{S}$ & $\mathrm{R}$ & $\mathrm{R}$ & $\mathrm{R}$ & & & 15 \\
\hline FA1C & Escherichia coli & hospital A/raw sewage & $\mathrm{R}$ & $\mathrm{R}$ & $\mathrm{R}$ & S & I & S & $\mathrm{R}$ & $\mathrm{R}$ & $\mathrm{R}$ & $\mathrm{R}$ & $\mathrm{R}$ & & & 123 \\
\hline FA3C & Klebsiella pneumoniae & hospital A/raw sewage & $\mathrm{R}$ & $\mathrm{R}$ & $\mathrm{S}$ & $\mathrm{S}$ & I & $\mathrm{R}$ & $\mathrm{R}$ & $\mathrm{R}$ & $\mathrm{R}$ & $\mathrm{R}$ & $\mathrm{R}$ & & & 15 \\
\hline FA4C & Escherichia coli & hospital A/raw sewage & $\mathrm{R}$ & $\mathrm{R}$ & $\mathrm{R}$ & S & $\mathrm{R}$ & $\mathrm{R}$ & I & $\mathrm{R}$ & $\mathrm{R}$ & $\mathrm{R}$ & $\mathrm{R}$ & & & 15 \\
\hline FA6C & Enterobacter sp. & hospital A/raw sewage & $\mathrm{R}$ & $\mathrm{R}$ & $\mathrm{R}$ & S & $\mathrm{R}$ & S & $S$ & $\mathrm{~S}$ & S & S & S & & & 65 \\
\hline FA7C & Escherichia coli & hospital A/raw sewage & $\mathrm{R}$ & $\mathrm{R}$ & $\mathrm{S}$ & $\mathrm{S}$ & $\mathrm{S}$ & $\mathrm{S}$ & $\mathrm{R}$ & $\mathrm{R}$ & $\mathrm{R}$ & $\mathrm{S}$ & $\mathrm{R}$ & & & 55 \\
\hline FA3M & Klebsiella pneumoniae & hospital A/raw sewage & $\mathrm{R}$ & $\mathrm{R}$ & $\mathrm{R}$ & $\mathrm{R}$ & $\mathrm{R}$ & $\mathrm{s}$ & I & $\mathrm{R}$ & $\mathrm{R}$ & $\mathrm{S}$ & $\mathrm{R}$ & 5 & & \\
\hline FA5M & Klebsiella pneumoniae & hospital A/raw sewage & $\mathrm{R}$ & $\mathrm{R}$ & $\mathrm{R}$ & $\mathrm{R}$ & $\mathrm{R}$ & $\mathrm{s}$ & I & $\mathrm{R}$ & $\mathrm{R}$ & $\mathrm{s}$ & $\mathrm{R}$ & 5 & & \\
\hline FA7M & Raoultella planticola & hospital A/raw sewage & $\mathrm{R}$ & $\mathrm{R}$ & $\mathrm{R}$ & $\mathrm{R}$ & $\mathrm{R}$ & $\mathrm{R}$ & $\mathrm{S}$ & $\mathrm{S}$ & $\mathrm{R}$ & $\mathrm{R}$ & $\mathrm{R}$ & 1 & 2 & 14 \\
\hline FA9M & Escherichia coli & hospital $\mathrm{A} /$ raw sewage & $\mathrm{R}$ & $\mathrm{R}$ & $\mathrm{R}$ & $\mathrm{R}$ & $\mathrm{R}$ & $\mathrm{R}$ & $\mathrm{R}$ & $\mathrm{R}$ & $\mathrm{R}$ & $\mathrm{R}$ & $\mathrm{R}$ & 5 & & 55 \\
\hline FA4M & Citrobacter braakii & hospital $\mathrm{A} /$ raw sewage & $\mathrm{R}$ & $\mathrm{R}$ & $\mathrm{R}$ & $\mathrm{R}$ & $\mathrm{R}$ & $\mathrm{R}$ & S & $\mathrm{S}$ & $\mathrm{R}$ & $\mathrm{R}$ & $\mathrm{R}$ & & 2 & \\
\hline ZA4M & Escherichia coli & hospital B/raw sewage & $\mathrm{R}$ & $\mathrm{R}$ & $\mathrm{R}$ & $\mathrm{R}$ & $\mathrm{R}$ & S & $\mathrm{S}$ & I & $\mathrm{R}$ & $\mathrm{R}$ & $\mathrm{R}$ & 5 & & 14 \\
\hline ZA3M & Escherichia coli & hospital B/raw sewage & $\mathrm{R}$ & $\mathrm{R}$ & $\mathrm{R}$ & $\mathrm{R}$ & I & S & $\mathrm{S}$ & $\mathrm{S}$ & S & $\mathrm{R}$ & $\mathrm{R}$ & 5 & & 15 \\
\hline ZA1M & Escherichia coli & hospital B/raw sewage & $\mathrm{R}$ & $\mathrm{R}$ & $\mathrm{R}$ & $\mathrm{R}$ & $\mathrm{R}$ & $\mathrm{R}$ & $\mathrm{R}$ & $\mathrm{R}$ & $\mathrm{R}$ & $\mathrm{R}$ & $\mathrm{R}$ & 5 & & 199 \\
\hline ZA10M & Escherichia coli & hospital B/raw sewage & $\mathrm{R}$ & $\mathrm{R}$ & $\mathrm{R}$ & $\mathrm{R}$ & $\mathrm{R}$ & $\mathrm{R}$ & $\mathrm{R}$ & $\mathrm{R}$ & $\mathrm{R}$ & $\mathrm{R}$ & $\mathrm{R}$ & 5 & & 199 \\
\hline ZA9M & Klebsiella pneumoniae & hospital B/raw sewage & $\mathrm{R}$ & $\mathrm{R}$ & $\mathrm{R}$ & $\mathrm{R}$ & $\mathrm{R}$ & S & $\mathrm{R}$ & $\mathrm{R}$ & $\mathrm{R}$ & $\mathrm{R}$ & $\mathrm{R}$ & & & 27 \\
\hline ZA7M & Citrobacter sp. & hospital B/raw sewage & $\mathrm{R}$ & $\mathrm{R}$ & $\mathrm{R}$ & $\mathrm{R}$ & $\mathrm{R}$ & $\mathrm{R}$ & $\mathrm{S}$ & $\mathrm{S}$ & $\mathrm{R}$ & $\mathrm{R}$ & $\mathrm{R}$ & 1 & 2 & 14 \\
\hline ZA5M & Escherichia coli & hospital B/raw sewage & $\mathrm{R}$ & $\mathrm{R}$ & $\mathrm{R}$ & $\mathrm{R}$ & $\mathrm{R}$ & $\mathrm{R}$ & $\mathrm{S}$ & $\mathrm{S}$ & S & $\mathrm{R}$ & $\mathrm{R}$ & 5 & & 123 \\
\hline ZA7C & Klebsiella pneumoniae & hospital B/raw sewage & $\mathrm{R}$ & $\mathrm{R}$ & $\mathrm{R}$ & $\mathrm{S}$ & $\mathrm{R}$ & $\mathrm{S}$ & $\mathrm{S}$ & $\mathrm{S}$ & $\mathrm{R}$ & $\mathrm{S}$ & $\mathrm{R}$ & & & \\
\hline ZA1C & Escherichia coli & hospital B/raw sewage & $\mathrm{R}$ & $\mathrm{R}$ & S & S & S & $\mathrm{R}$ & $\mathrm{R}$ & $\mathrm{S}$ & $\mathrm{R}$ & S & $\mathrm{R}$ & & & 55 \\
\hline ZA2C & Escherichia coli & hospital B/raw sewage & $\mathrm{R}$ & $\mathrm{R}$ & $\mathrm{R}$ & S & I & S & I & $\mathrm{S}$ & S & $\mathrm{R}$ & $\mathrm{R}$ & & & 55 \\
\hline ZA4C & Escherichia coli & hospital B/raw sewage & $\mathrm{R}$ & $\mathrm{R}$ & $\mathrm{R}$ & $\mathrm{s}$ & $\mathrm{R}$ & $\mathrm{R}$ & $\mathrm{R}$ & $\mathrm{R}$ & $\mathrm{R}$ & I & $\mathrm{S}$ & & & 55 \\
\hline ZA5C & Escherichia coli & hospital B/raw sewage & $\mathrm{R}$ & $\mathrm{R}$ & $\mathrm{S}$ & $\mathrm{S}$ & $\mathrm{S}$ & $\mathrm{R}$ & $\mathrm{R}$ & $\mathrm{S}$ & $\mathrm{R}$ & $\mathrm{S}$ & $\mathrm{R}$ & & & 55 \\
\hline ZA6C & Escherichia coli & hospital B/raw sewage & $\mathrm{R}$ & $\mathrm{R}$ & S & S & I & $\mathrm{R}$ & S & $\mathrm{S}$ & $\mathrm{R}$ & $\mathrm{R}$ & $\mathrm{R}$ & & & 55 \\
\hline RA2M & Escherichia coli & hospital C/raw sewage & $\mathrm{R}$ & $\mathrm{R}$ & $\mathrm{R}$ & $\mathrm{R}$ & $\mathrm{R}$ & $\mathrm{R}$ & $\mathrm{R}$ & $\mathrm{R}$ & $\mathrm{R}$ & $\mathrm{R}$ & $\mathrm{R}$ & 5 & & 55 \\
\hline RA1M & Enterobacter kobei & hospital C/raw sewage & $\mathrm{R}$ & $\mathrm{R}$ & $\mathrm{R}$ & $\mathrm{R}$ & $\mathrm{R}$ & S & S & $\mathrm{S}$ & S & S & S & & 2 & 9 \\
\hline RA13C & Raoultella planticola & hospital C/raw sewage & $\mathrm{R}$ & $\mathrm{R}$ & S & $\mathrm{R}$ & $\mathrm{R}$ & $\mathrm{R}$ & $\mathrm{S}$ & $\mathrm{S}$ & S & S & $\mathrm{R}$ & & 2 & \\
\hline RA14M & Klebsiella pneumoniae & hospital C/raw sewage & $\mathrm{R}$ & $\mathrm{R}$ & $\mathrm{R}$ & $\mathrm{R}$ & $\mathrm{R}$ & $\mathrm{S}$ & I & $\mathrm{R}$ & $\mathrm{R}$ & $\mathrm{R}$ & $\mathrm{S}$ & & 2 & \\
\hline RA11M & Klebsiella pneumoniae & hospital C/raw sewage & $\mathrm{R}$ & $\mathrm{R}$ & $\mathrm{R}$ & $\mathrm{R}$ & $\mathrm{R}$ & $\mathrm{R}$ & $\mathrm{S}$ & $\mathrm{R}$ & S & $\mathrm{R}$ & $\mathrm{R}$ & & 2 & 65 \\
\hline
\end{tabular}


Table 1. Cont

\begin{tabular}{|c|c|c|c|c|c|c|c|c|c|c|c|c|c|c|c|c|}
\hline Isolation No. ${ }^{a}$ & Species & Isolation Source & $\mathrm{AMP}^{\mathrm{b}}$ & CTX & FOX & MEM & AMC & $\mathrm{CN}$ & $\mathrm{S}$ & $\mathrm{C}$ & TET & CIP & SXT & NDM & KPC & CTX-M \\
\hline RA6C & Klebsiella pneumoniae & hospital C/raw sewage & $\mathrm{R}$ & $\mathrm{R}$ & $\mathrm{R}$ & $\mathrm{R}$ & $\mathrm{R}$ & $\mathrm{R}$ & $\mathrm{S}$ & $\mathrm{S}$ & $\mathrm{S}$ & $\mathrm{S}$ & $\mathrm{R}$ & & 2 & \\
\hline RA5C & Escherichia coli & hospital C/raw sewage & $\mathrm{R}$ & $\mathrm{R}$ & S & S & I & $S$ & $\mathrm{R}$ & $\mathrm{R}$ & $\mathrm{R}$ & S & $\mathrm{R}$ & & & 55 \\
\hline RA4C & Escherichia coli & hospital C/raw sewage & $\mathrm{R}$ & $\mathrm{R}$ & $\mathrm{S}$ & $\mathrm{R}$ & I & $\mathrm{R}$ & I & $\mathrm{S}$ & $\mathrm{R}$ & $\mathrm{R}$ & $\mathrm{R}$ & 1 & & 14 \\
\hline RA2C & Escherichia coli & hospital C/raw sewage & $\mathrm{R}$ & $\mathrm{R}$ & $\mathrm{S}$ & S & $\mathrm{R}$ & $\mathrm{S}$ & $\mathrm{R}$ & I & $\mathrm{R}$ & $\mathrm{R}$ & $\mathrm{R}$ & & & 55 \\
\hline RA1C & Escherichia coli & hospital C/raw sewage & $\mathrm{R}$ & $\mathrm{R}$ & $\mathrm{S}$ & $\mathrm{s}$ & $\mathrm{R}$ & $\mathrm{S}$ & I & $\mathrm{R}$ & $\mathrm{R}$ & $\mathrm{S}$ & $\mathrm{S}$ & & & 55 \\
\hline RA10M & Enterobacter sp. & hospital C/raw sewage & $\mathrm{R}$ & $\mathrm{R}$ & $\mathrm{R}$ & $\mathrm{R}$ & $\mathrm{R}$ & $S$ & $\mathrm{~S}$ & $\mathrm{~S}$ & $\mathrm{~S}$ & $\mathrm{~S}$ & $\mathrm{~S}$ & & 2 & 55 \\
\hline RA7M & Escherichia coli & hospital C/raw sewage & $\mathrm{R}$ & $\mathrm{R}$ & $\mathrm{R}$ & $\mathrm{R}$ & $\mathrm{R}$ & $\mathrm{R}$ & $\mathrm{R}$ & $\mathrm{R}$ & $\mathrm{R}$ & $\mathrm{R}$ & $\mathrm{R}$ & 5 & & + \\
\hline RA12C & Escherichia coli & hospital C/raw sewage & $\mathrm{R}$ & $\mathrm{R}$ & $\mathrm{S}$ & $\mathrm{R}$ & $\mathrm{R}$ & $\mathrm{S}$ & $\mathrm{S}$ & $\mathrm{S}$ & $\mathrm{S}$ & $\mathrm{R}$ & $\mathrm{S}$ & 1 & & 55 \\
\hline RA11C & Escherichia coli & hospital C/raw sewage & $\mathrm{R}$ & $\mathrm{R}$ & $\mathrm{S}$ & $\mathrm{R}$ & S & $\mathrm{S}$ & $\mathrm{S}$ & $\mathrm{S}$ & $\mathrm{S}$ & $\mathrm{R}$ & $\mathrm{S}$ & 1 & & \\
\hline RA8C & Klebsiella pneumoniae & hospital C/raw sewage & $\mathrm{R}$ & $\mathrm{R}$ & $\mathrm{R}$ & $\mathrm{S}$ & $\mathrm{R}$ & $\mathrm{R}$ & $\mathrm{S}$ & $\mathrm{R}$ & $\mathrm{R}$ & $\mathrm{R}$ & $\mathrm{R}$ & & & 27 \\
\hline RA7C & Escherichia coli & hospital C/raw sewage & $\mathrm{R}$ & $\mathrm{R}$ & $\mathrm{S}$ & $\mathrm{S}$ & $\mathrm{R}$ & $\mathrm{R}$ & $\mathrm{S}$ & $\mathrm{S}$ & $\mathrm{R}$ & $\mathrm{R}$ & $\mathrm{R}$ & & & 15 \\
\hline RA8M & Escherichia coli & hospital C/raw sewage & $\mathrm{R}$ & $\mathrm{R}$ & $\mathrm{R}$ & $\mathrm{R}$ & $\mathrm{R}$ & $\mathrm{R}$ & $\mathrm{R}$ & $\mathrm{R}$ & $\mathrm{R}$ & $\mathrm{S}$ & $\mathrm{R}$ & & & 55 \\
\hline RB1C & Escherichia coli & hospital C/effluent & $\mathrm{R}$ & $\mathrm{R}$ & $\mathrm{S}$ & $\mathrm{S}$ & I & $\mathrm{R}$ & $\mathrm{R}$ & $\mathrm{R}$ & $\mathrm{R}$ & $\mathrm{R}$ & $\mathrm{R}$ & & & 55 \\
\hline RB7C & Escherichia coli & hospital C/effluent & $\mathrm{R}$ & $\mathrm{R}$ & I & $\mathrm{R}$ & S & $S$ & I & $\mathrm{S}$ & S & $\mathrm{R}$ & $\mathrm{R}$ & & 2 & 14 \\
\hline RB6C & Escherichia coli & hospital C/effluent & $\mathrm{R}$ & $\mathrm{R}$ & $\mathrm{S}$ & $\mathrm{S}$ & I & $\mathrm{R}$ & $\mathrm{R}$ & $\mathrm{R}$ & $\mathrm{R}$ & $\mathrm{R}$ & $\mathrm{R}$ & & & 55 \\
\hline RB4C & Escherichia coli & hospital C/effluent & $\mathrm{R}$ & $\mathrm{R}$ & $\mathrm{S}$ & $\mathrm{S}$ & $\mathrm{R}$ & $\mathrm{S}$ & $\mathrm{S}$ & $\mathrm{R}$ & $\mathrm{R}$ & $\mathrm{S}$ & $\mathrm{S}$ & & & 55 \\
\hline RB3M & Klebsiella pneumoniae & hospital C/effluent & $\mathrm{R}$ & $\mathrm{R}$ & $\mathrm{R}$ & $\mathrm{R}$ & $\mathrm{R}$ & $\mathrm{R}$ & $\mathrm{R}$ & $\mathrm{R}$ & $\mathrm{R}$ & $\mathrm{R}$ & $\mathrm{R}$ & 5 & & 55 \\
\hline RB6M & Klebsiella variicola & hospital C/effluent & $\mathrm{R}$ & $\mathrm{R}$ & $\mathrm{R}$ & $\mathrm{R}$ & $\mathrm{R}$ & $\mathrm{R}$ & $\mathrm{R}$ & $\mathrm{R}$ & $\mathrm{R}$ & S & $\mathrm{R}$ & 5 & & 55 \\
\hline CS3C & Escherichia coli & river water & $\mathrm{R}$ & $\mathrm{R}$ & S & S & S & S & S & $\mathrm{S}$ & S & $\mathrm{R}$ & $\mathrm{R}$ & & & 14 \\
\hline CS4C & Enterobacter tabaci & river water & $\mathrm{R}$ & $\mathrm{R}$ & $\mathrm{R}$ & S & $\mathrm{R}$ & $S$ & $\mathrm{~S}$ & $\mathrm{~S}$ & $\mathrm{~S}$ & $\mathrm{~S}$ & S & & & + \\
\hline CS8C & Escherichia coli & river water & $\mathrm{R}$ & $\mathrm{R}$ & $\mathrm{S}$ & $\mathrm{s}$ & $\mathrm{R}$ & $\mathrm{S}$ & $\mathrm{R}$ & $\mathrm{S}$ & $\mathrm{R}$ & $\mathrm{R}$ & $\mathrm{R}$ & & & 55 \\
\hline TX1C & Klebsiella variicola & river water & $\mathrm{R}$ & $\mathrm{R}$ & $\mathrm{R}$ & $\mathrm{S}$ & $\mathrm{R}$ & $\mathrm{S}$ & $\mathrm{S}$ & $\mathrm{S}$ & $\mathrm{R}$ & $\mathrm{S}$ & $\mathrm{R}$ & & & + \\
\hline $\mathrm{TX} 2 \mathrm{C}$ & Klebsiella pneumoniae & river water & $\mathrm{R}$ & $\mathrm{R}$ & S & S & S & $\mathrm{S}$ & $\mathrm{S}$ & $\mathrm{R}$ & $\mathrm{R}$ & S & $\mathrm{R}$ & & & 27 \\
\hline TX5C & Klebsiella pneumoniae & river water & $\mathrm{R}$ & $\mathrm{R}$ & $\mathrm{R}$ & $\mathrm{s}$ & $\mathrm{R}$ & $\mathrm{S}$ & $\mathrm{R}$ & $\mathrm{S}$ & $\mathrm{R}$ & $\mathrm{S}$ & $\mathrm{R}$ & & & + \\
\hline TS1C & Klebsiella pneumoniae & river water & $\mathrm{R}$ & $\mathrm{R}$ & $\mathrm{R}$ & $\mathrm{S}$ & S & $S$ & $\mathrm{~S}$ & $\mathrm{~S}$ & $\mathrm{~S}$ & $\mathrm{~S}$ & $\mathrm{~S}$ & & & + \\
\hline TS11C & Escherichia coli & river water & $\mathrm{R}$ & $\mathrm{R}$ & $\mathrm{S}$ & $\mathrm{S}$ & $\mathrm{S}$ & $\mathrm{S}$ & $\mathrm{s}$ & $\mathrm{S}$ & $\mathrm{R}$ & $\mathrm{s}$ & $\mathrm{S}$ & & & \\
\hline TS3M & Escherichia coli & river water & $\mathrm{R}$ & $\mathrm{R}$ & $\mathrm{R}$ & $\mathrm{R}$ & $\mathrm{R}$ & $\mathrm{R}$ & $\mathrm{R}$ & $\mathrm{R}$ & $\mathrm{R}$ & $\mathrm{R}$ & $\mathrm{R}$ & 5 & & \\
\hline TS4M & Klebsiella pneumoniae & river water & $\mathrm{R}$ & $\mathrm{R}$ & $\mathrm{R}$ & $\mathrm{R}$ & $\mathrm{R}$ & $\mathrm{R}$ & $\mathrm{R}$ & $\mathrm{R}$ & $\mathrm{R}$ & $\mathrm{R}$ & $\mathrm{R}$ & 5 & & \\
\hline CX1M & Escherichia coli & river water & $\mathrm{R}$ & $\mathrm{R}$ & $\mathrm{R}$ & $\mathrm{R}$ & $\mathrm{R}$ & $\mathrm{R}$ & $\mathrm{R}$ & $\mathrm{S}$ & $\mathrm{R}$ & $\mathrm{R}$ & S & 1 & & \\
\hline TX5M & Citrobacter freundii & river water & $\mathrm{R}$ & $\mathrm{R}$ & $\mathrm{R}$ & $\mathrm{R}$ & $\mathrm{R}$ & $\mathrm{R}$ & $\mathrm{R}$ & $\mathrm{R}$ & $\mathrm{R}$ & $\mathrm{R}$ & $\mathrm{R}$ & 5 & & \\
\hline TX6M & Escherichia coli & river water & $\mathrm{R}$ & $\mathrm{R}$ & $\mathrm{R}$ & $\mathrm{R}$ & $\mathrm{R}$ & $\mathrm{R}$ & I & $\mathrm{R}$ & $\mathrm{R}$ & S & $\mathrm{R}$ & & & \\
\hline
\end{tabular}

${ }^{a}$ Multidrug-resistant strains are highlighted in orange. ${ }^{\mathrm{b}}$ ampicillin (AMP), amoxicillin-clavulanic acid (AMC), ciprofloxacin (CIP), tetracycline (TET), gentamicin (CN), chloramphenicol (C), streptomycin (S), cefoxitin (FOX), cefotaxime (CTX), meropenem (MEM), and trimethoprim/sulfamethoxazole (SXT). Antibiotic susceptibility is depicted with S for susceptible, I for intermediate resistant and $\mathrm{R}$ for resistant (highlighted in green). 
Table 2. Acinetobacter strains isolated from aquatic environments.

\begin{tabular}{|c|c|c|c|c|c|c|c|c|c|c|c|}
\hline Isolation No. ${ }^{a}$ & Isolation Source & $\mathrm{CTX}^{\mathrm{b}}$ & FOX & MEM & $\mathrm{CN}$ & TET & CIP & SXT & NDM & OXA & CTX-M \\
\hline FA2C & hospital A/raw sewage & $\mathrm{R}$ & $\mathrm{R}$ & $S$ & $S$ & $S$ & $S$ & S & & & 65 \\
\hline FA2M & hospital A/raw sewage & $\mathrm{R}$ & $\mathrm{R}$ & $\mathrm{R}$ & $\mathrm{R}$ & $\mathrm{R}$ & $\mathrm{R}$ & $\mathrm{R}$ & 1 & & \\
\hline ZA2M & hospital B/raw sewage & $\mathrm{R}$ & $\mathrm{R}$ & $\mathrm{R}$ & $\mathrm{R}$ & $\mathrm{R}$ & I & $\mathrm{R}$ & 1 & 58 & \\
\hline ZA6M & hospital B/raw sewage & $\mathrm{R}$ & $\mathrm{R}$ & $\mathrm{R}$ & $\mathrm{R}$ & $\mathrm{R}$ & $S$ & $\mathrm{R}$ & 1 & & \\
\hline ZA8M & hospital B/raw sewage & $\mathrm{R}$ & S & S & $\mathrm{R}$ & S & S & I & & & \\
\hline ZA8C & hospital B/raw sewage & $\mathrm{R}$ & $\mathrm{R}$ & $S$ & S & S & $S$ & $S$ & & & 55 \\
\hline RA5M & hospital C/raw sewage & $\mathrm{R}$ & $\mathrm{R}$ & $\mathrm{R}$ & S & $\mathrm{R}$ & $S$ & $\mathrm{R}$ & 1 & & \\
\hline RA9M & hospital C/raw sewage & $\mathrm{R}$ & $\mathrm{R}$ & $\mathrm{R}$ & $\mathrm{R}$ & $\mathrm{R}$ & $\mathrm{R}$ & $\mathrm{R}$ & 1 & 58 & \\
\hline RA12M & hospital C/raw sewage & $\mathrm{R}$ & $\mathrm{R}$ & $\mathrm{R}$ & $\mathrm{R}$ & $\mathrm{R}$ & I & $\mathrm{R}$ & 5 & 58 & 3 \\
\hline RA3M & hospital C/raw sewage & $\mathrm{R}$ & $\mathrm{R}$ & $\mathrm{R}$ & S & $\mathrm{R}$ & I & $\mathrm{R}$ & 1 & 58 & \\
\hline RA4M & hospital C/raw sewage & $\mathrm{R}$ & $\mathrm{R}$ & $\mathrm{R}$ & $\mathrm{R}$ & $\mathrm{R}$ & I & $\mathrm{R}$ & 1 & 58 & \\
\hline RB4M & hospital C/effluent & $\mathrm{R}$ & $\mathrm{R}$ & $\mathrm{R}$ & $\mathrm{R}$ & $\mathrm{R}$ & S & $\mathrm{R}$ & 1 & & \\
\hline RB5C & hospital C/effluent & $\mathrm{R}$ & $\mathrm{R}$ & $\mathrm{R}$ & $\mathrm{R}$ & $\mathrm{R}$ & S & $\mathrm{R}$ & 1 & & 55 \\
\hline RB1M & hospital C/effluent & $\mathrm{R}$ & $\mathrm{R}$ & $\mathrm{R}$ & $\mathrm{R}$ & $\mathrm{R}$ & I & $\mathrm{R}$ & & & 55 \\
\hline RB2M & hospital C/effluent & $\mathrm{R}$ & $\mathrm{R}$ & $\mathrm{R}$ & $\mathrm{R}$ & $\mathrm{R}$ & $S$ & $\mathrm{R}$ & 1 & 58 & 55 \\
\hline RB5M & hospital C/effluent & $\mathrm{R}$ & $\mathrm{R}$ & $\mathrm{R}$ & S & $\mathrm{R}$ & I & I & 1 & 58 & 3 \\
\hline CX9C & river water & $\mathrm{R}$ & I & S & $S$ & S & S & S & & & \\
\hline CX11C & river water & $\mathrm{R}$ & S & S & S & $\mathrm{R}$ & S & S & & & \\
\hline TX3C & river water & $\mathrm{R}$ & $\mathrm{R}$ & S & S & $\mathrm{R}$ & S & $\mathrm{R}$ & & & \\
\hline CS1M & river water & $\mathrm{R}$ & $\mathrm{R}$ & $\mathrm{R}$ & S & S & $S$ & S & 1 & & \\
\hline
\end{tabular}

${ }^{a}$ Multidrug-resistant strains are highlighted in orange. ${ }^{b}$ cefotaxime (CTX), cefoxitin (FOX), meropenem (MEM), gentamicin (CN), tetracycline (TET), ciprofloxacin (CIP), and trimethoprim/sulfamethoxazole (SXT). Antibiotic susceptibility is depicted with $\mathrm{S}$ for susceptible, I for intermediate resistant and $\mathrm{R}$ for resistant (highlighted in green).

\section{Discussion}

Aquatic environments are significant reservoirs of antibiotic-resistant determinants. They also serve as a vehicle by which ARGs or ARB could be disseminated from one ecosystem to another, thereby increasing the risk of infection with MDR bacteria outside the hospital [31]. Among the MDR bacteria, the widespread nature of $\mathrm{CPB}$ posed a high risk to the global public health. In this work, CPB were readily detected in raw sewage, treated effluent and river water samples. NDM was identified to be the most prevalent carbapenemase and it was commonly harbored in Enterobacteriaceae, as well as in some non-Enterobacteriaceae families (Acinetobacter spp., Aeromonas spp., and Pseudomonas spp.). Our results are consistent with previous studies, showing that $b l a_{\mathrm{NDM}}$ has occurred in many unrelated species and is spreading rapidly in different environmental compartments $[15,18,32]$. The genus Acinetobacter, which is widespread in nature and particularly abundant in wastewater, has been recognized in recent years as a universal threat to public health [10]. The high prevalence of bla $a_{\mathrm{NDM}}$ in Acinetobacter species is worrisome, because these organisms may serve as potential reservoirs and propagate the $b l a_{\text {NDM }}$ gene through highly transmissible genetic elements, ultimately increasing the emergence of new population of carbapenem-resistant isolates. Multiple variants of $b l a_{\mathrm{NDM}}$ have been identified in clinical and environmental samples [33]. Our results further supplement previous studies that show NDM-1 to be widely distributed in wastewater in China [20,34,35]. We also found that NDM-5, which exhibits increased resistance to carbapenems and broad-spectrum cephalosporins [33], was also highly prevalent in the collected samples. The wide dissemination of $b l a_{\mathrm{NDM}-5}$ in the environment raises a serious public health concern.

KPC-producing isolates were frequently recovered from environmental samples in China $[19,24,36,37]$. Aeromonas spp. are among the dominant genera in wastewater communities and members of this genus are opportunistic pathogens of humans and animals [38]. Despite several studies reporting the emergence of multidrug-resistant and/or $\beta$-lactamase-producing Aeromonas isolates [38,39], a high prevalence of bla $a_{\mathrm{KPC}-2}$ in Aeromonas in this study was still quite unexpected. A recent study also revealed two Aeromonas isolates carrying $b l a_{\mathrm{KPC}-2}$ from urban wastewater treatment plant (WWTP) effluents in Japan [40]. The literature data and our findings suggest that Aeromonas spp. may be involved in the maintenance and dissemination of the $b l a_{\mathrm{KPC}-2}$ gene in the environment.

The occurrence of strains co-producing two different carbapenemase families is increasingly frequent in environmental matrices. It was reported that $b l a_{\mathrm{KPC}}$ and $b l a_{\mathrm{NDM}-1}$ coexisted in two 
P. aeruginosa and one C. freundii from hospital effluents in Singapore [18]. One Citrobacter braakii strain was identified co-harboring $b l a_{\mathrm{NDM}-4}$ and $b l a_{\mathrm{OXA}-48}$ from hospital sewage in northern India [15]. An Acinetobacter towneri strain was identified co-harboring $b l a_{\mathrm{NDM}-1}$ and $b l a_{\mathrm{OXA}-58}$ from hospital sewage from one of our sampling sites (hospital A) [23]. In our work, the coexistence of $b l a_{\mathrm{NDM}-1}$ and $b l a_{\mathrm{OXA}-58}$ genes was identified in six Acinetobacter isolates, of which five were from hospital $\mathrm{C}$ and one from hospital B. Additionally, one Acinetobacter strain was found to co-harbor the $b l a_{\mathrm{NDM}-5}$ and $b l a_{\mathrm{OXA}-58}$ genes. These findings indicate that Acinetobacter strains coproducing NDM and OXA-58 were kind of prevalent in this region. The coexistence of NDM-1 and KPC-2 in environmental Enterobacteriaceae isolates, although not very common, was alarming and more research is needed to further elucidate the dissemination of these clinically significant antibiotic resistance genes.

Analysis of the antimicrobial resistance profiles of Enterobacteriaceae and Acinetobacter isolates obtained in this study revealed a high prevalence of resistance to antimicrobial agents, and that MDR strains were frequently observed. In addition to $\beta$-lactam resistance, a relatively high percentage of resistance to trimethoprim/sulfamethoxazole and tetracycline was also identified. This finding was consistent with previous studies that tetracycline and sulfonamide resistance genes were widely found in aquaculture environments $[15,41]$. Notably, most carbapenem-resistant strains were cross-resistant to other classes of clinically important antibiotics, including quinolones (ciprofloxacin) and aminoglycosides (gentamicin).

There is a growing number of studies reporting that, although WWTPs can efficiently remove many ARB, ARB or ARGs are still detectable at high concentrations in treated effluents [35,36,40]. In this study, we found that several carbapenemase-producing isolates persisted in the disinfected effluents from hospital WWTPs. We also detected carbapenemase producers in the receiving rivers which are affected by hospital WWTPs. These findings suggest that WWTP effluents could be a potential source for the dissemination of clinically important antibiotic resistance genes, such as carbapenemase genes, after their discharge into aquatic environments. The presence of a number of MDR opportunistic pathogens, such as E. coli, K. pneumoniae, Enterobacter spp. and Acinetobacter spp. in WWTP effluents and riverine environments represents an emerging public health issue. More efforts should be made to improve the elimination of antimicrobial resistant microorganisms from hospital sewage.

\section{Conclusions}

This study highlights the prevalence of ESBL- and carbapenemase-resistant Enterobacteriaceae and non-Enterobacteriaceae species (such as Acinetobacter and Aeromonas) in hospital sewage systems and receiving waters in China. Among these resistant isolates, a large proportion of the Enterobacteriaceae and Acinetobacter strains exhibited a multidrug resistance phenotype. The detection of clinically significant multidrug-resistant strains in the aquatic environment could have serious public health implications, which demands more attention. Furthermore, our findings also reinforce the idea that hospital WWTP effluent could be an important source of ARB propagation into the environment. Future projects should be conducted in more water bodies across the country to obtain a better picture of the situation. Superior disinfection techniques are expected to improve the elimination of ARB during wastewater treatment, and closer monitoring of the quality of treated effluents is needed to minimize the dissemination of antimicrobial resistance into the receiving environment.

Supplementary Materials: The following are available online at http://www.mdpi.com/1660-4601/17/4/1183/s1, Table S1: Primers used in this study.

Author Contributions: Conceptualization, L.Z. and Y.L.; methodology, X.M.; software, L.L. and N.H.; formal analysis, J.D. and Z.T.; resources, R.Z.; writing-original draft preparation, Y.L.; writing-review and editing, L.Z. All authors have read and agreed to the published version of the manuscript.

Funding: This work was supported by National Natural Science Foundation of China (31900125), the Scienceand Technology Strategic Cooperation Programs of Luzhou Municipal People's Government and Southwest Medical University (2019LZXNYDJ47), Natural Science Foundation of Southwest Medical University (2018-ZRZD-011), and Undergraduate Innovation and Entrepreneurship Project (2019492). The funders had no role in study design, data collection and interpretation, or the decision to submit the work for publication. 
Conflicts of Interest: The authors declare no conflict of interest.

\section{References}

1. Blair, J.M.; Webber, M.A.; Baylay, A.J.; Ogbolu, D.O.; Piddock, L.J. Molecular mechanisms of antibiotic resistance. Nat. Rev. Microbiol. 2015, 13, 42-51. [CrossRef]

2. Pitout, J.D.D.; Laupland, K.B. Extended-spectrum $\beta$-lactamase-producing Enterobacteriaceae: An emerging public-health concern. Lancet Infect. Dis. 2008, 8, 159-166. [CrossRef]

3. Bonomo, R.A. Beta-Lactamases: A Focus on Current Challenges. Cold Spring Harb. Perspect. Med. $2017,7$. [CrossRef] [PubMed]

4. Meletis, G. Carbapenem resistance: Overview of the problem and future perspectives. Ther. Adv. Infect. Dis. 2016, 3, 15-21. [CrossRef] [PubMed]

5. Bush, K. Past and Present Perspectives on $\beta$-Lactamases. Antimicrob. Agents Chemother. 2018, 62, e01076-18. [CrossRef]

6. Schwaber, M.J.; Klarfeld-Lidji, S.; Navon-Venezia, S.; Schwartz, D.; Leavitt, A.; Carmeli, Y. Predictors of Carbapenem-Resistant Klebsiella pneumoniae Acquisition among Hospitalized Adults and Effect of Acquisition on Mortality. Antimicrob. Agents Chemother. 2008, 52, 1028-1033. [CrossRef]

7. Martin, A.; Fahrbach, K.; Zhao, Q.; Lodise, T. Association Between Carbapenem Resistance and Mortality Among Adult, Hospitalized Patients With Serious Infections Due to Enterobacteriaceae: Results of a Systematic Literature Review and Meta-analysis. Open Forum Infect. Dis. 2018, 5, ofy150. [CrossRef]

8. Potter, R.F.; D'Souza, A.W.; Dantas, G. The rapid spread of carbapenem-resistant Enterobacteriaceae. Drug Resist. Updated 2016, 29, 30-46. [CrossRef]

9. Nordmann, P.; Dortet, L.; Poirel, L. Carbapenem resistance in Enterobacteriaceae: here is the storm! Trends Mol. Med. 2012, 18, 263-272. [CrossRef] [PubMed]

10. Maravic, A.; Skocibusic, M.; Fredotovic, Z.; Samanic, I.; Cvjetan, S.; Knezovic, M.; Puizina, J. Urban riverine environment is a source of multidrug-resistant and ESBL-producing clinically important Acinetobacter spp. Environ. Sci. Pollut. Res. Int. 2016, 23, 3525-3535. [CrossRef]

11. Rozwandowicz, M.; Brouwer, M.S.M.; Fischer, J.; Wagenaar, J.A.; Gonzalez-Zorn, B.; Guerra, B.; Mevius, D.J.; Hordijk, J. Plasmids carrying antimicrobial resistance genes in Enterobacteriaceae. J. Antimicrob. Chemother. 2018, 73, 1121-1137. [CrossRef] [PubMed]

12. Karkman, A.; Do, T.T.; Walsh, F.; Virta, M.P.J. Antibiotic-Resistance Genes in Waste Water. Trends Microbiol. 2018, 26, 220-228. [CrossRef] [PubMed]

13. Weingarten, R.A.; Johnson, R.C.; Conlan, S.; Ramsburg, A.M.; Dekker, J.P.; Lau, A.F.; Khil, P.; Odom, R.T.; Deming, C.; Park, M.; et al. Genomic Analysis of Hospital Plumbing Reveals Diverse Reservoir of Bacterial Plasmids Conferring Carbapenem Resistance. mBio 2018, 9, e02011-e02017. [CrossRef] [PubMed]

14. Lamba, M.; Graham, D.W.; Ahammad, S.Z. Hospital Wastewater Releases of Carbapenem-Resistance Pathogens and Genes in Urban India. Environ. Sci. Technol. 2017, 51, 13906-13912. [CrossRef]

15. Parvez, S.; Khan, A.U. Hospital sewage water: A reservoir for variants of New Delhi metallo-beta-lactamase (NDM)- and extended-spectrum beta-lactamase (ESBL)-producing Enterobacteriaceae. Int. J. Antimicrob. Agents 2018, 51, 82-88. [CrossRef] [PubMed]

16. Nasri, E.; Subirats, J.; Sanchez-Melsio, A.; Mansour, H.B.; Borrego, C.M.; Balcazar, J.L. Abundance of carbapenemase genes (blaKPC, blaNDM and blaOXA-48) in wastewater effluents from Tunisian hospitals. Environ. Pollut. 2017, 229, 371-374. [CrossRef] [PubMed]

17. Cahill, N.; O’Connor, L.; Mahon, B.; Varley, A.; McGrath, E.; Ryan, P.; Cormican, M.; Brehony, C.; Jolley, K.A.; Maiden, M.C.; et al. Hospital effluent: A reservoir for carbapenemase-producing Enterobacterales? Sci. Total Environ. 2019, 672, 618-624. [CrossRef] [PubMed]

18. Haller, L.; Chen, H.; Ng, C.; Le, T.H.; Koh, T.H.; Barkham, T.; Sobsey, M.; Gin, K.Y. Occurrence and characteristics of extended-spectrum beta-lactamase- and carbapenemase- producing bacteria from hospital effluents in Singapore. Sci. Total Environ. 2018, 615, 1119-1125. [CrossRef]

19. Zhang, X.; Lü, X.; Zong, Z. Enterobacteriaceae producing the KPC-2 carbapenemase from hospital sewage. Diagn. Microbiol. Infect. Dis. 2012, 73, 204-206. [CrossRef] 
20. Zhang, C.; Qiu, S.; Wang, Y.; Qi, L.; Hao, R.; Liu, X.; Shi, Y.; Hu, X.; An, D.; Li, Z.; et al. Higher isolation of NDM-1 producing Acinetobacter baumannii from the sewage of the hospitals in Beijing. PLoS ONE 2014, 8, e64857. [CrossRef]

21. Jin, L.; Wang, R.; Wang, X.; Wang, Q.; Zhang, Y.; Yin, Y.; Wang, H. Emergence of mcr-1 and carbapenemase genes in hospital sewage water in Beijing, China. J. Antimicrob. Chemother. 2018, 73, 84-87. [CrossRef] [PubMed]

22. Zong, Z.; Zhang, X. blaNDM-1-carrying Acinetobacter johnsonii detected in hospital sewage. J. Antimicrob. Chemother. 2013, 68, 1007-1010. [CrossRef] [PubMed]

23. Jiang, N.; Zhang, X.; Zhou, Y.; Zhang, Z.; Zheng, X. Whole-genome sequencing of an NDM-1- and OXA-58-producing Acinetobacter towneri isolate from hospital sewage in Sichuan Province, China. J. Glob. Antimicrob. Resist. 2019, 16, 4-5. [CrossRef] [PubMed]

24. Xu, H.; Wang, X.; Yu, X.; Zhang, J.; Guo, L.; Huang, C.; Jiang, X.; Li, X.; Feng, Y.; Zheng, B. First detection and genomics analysis of KPC-2-producing Citrobacter isolates from river sediments. Environ. Pollut. 2018, 235, 931-937. [CrossRef] [PubMed]

25. Tafoukt, R.; Touati, A.; Leangapichart, T.; Bakour, S.; Rolain, J.M. Characterization of OXA-48-like-producing Enterobacteriaceae isolated from river water in Algeria. Water Res. 2017, 120, 185-189. [CrossRef] [PubMed]

26. Di, D.Y.W.; Jang, J.; Unno, T.; Hur, H.-G. Emergence of Klebsiella variicola positive for NDM-9, a variant of New Delhi metallo- $\beta$-lactamase, in an urban river in South Korea. J. Antimicrob. Chemother. 2017, 72, 1063-1067. [CrossRef] [PubMed]

27. Azuma, T.; Otomo, K.; Kunitou, M.; Shimizu, M.; Hosomaru, K.; Mikata, S.; Ishida, M.; Hisamatsu, K.; Yunoki, A.; Mino, Y.; et al. Environmental fate of pharmaceutical compounds and antimicrobial-resistant bacteria in hospital effluents, and contributions to pollutant loads in the surface waters in Japan. Sci. Total Environ. 2019, 657, 476-484. [CrossRef] [PubMed]

28. Khan, F.A.; Hellmark, B.; Ehricht, R.; Söderquist, B.; Jass, J. Related carbapenemase-producing Klebsiella isolates detected in both a hospital and associated aquatic environment in Sweden. Eur. J. Clin. Microbiol. Infect. Dis. 2018, 37, 2241-2251. [CrossRef] [PubMed]

29. Lane, D.J. 16S/23S rRNA sequencing. In Nucleic Acid Techniques in Bacterial Systematics; Wiley: New York, NY, USA, 1991; pp. 115-175.

30. CLSI. Performance Standards for Antimicrobial Susceptibility Testing, 29th ed.; CLSI Supplement M100; Clinical and Laboratory Standards Institute: Wayne, PA, USA, 2019.

31. Sivalingam, P.; Pote, J.; Prabakar, K. Environmental Prevalence of Carbapenem Resistance Enterobacteriaceae (CRE) in a Tropical Ecosystem in India: Human Health Perspectives and Future Directives. Pathogens 2019, 8, 174. [CrossRef] [PubMed]

32. Marathe, N.P.; Berglund, F.; Razavi, M.; Pal, C.; Droge, J.; Samant, S.; Kristiansson, E.; Larsson, D.G.J. Sewage effluent from an Indian hospital harbors novel carbapenemases and integron-borne antibiotic resistance genes. Microbiome 2019, 7, 97. [CrossRef]

33. Wu, W.; Feng, Y.; Tang, G.; Qiao, F.; McNally, A.; Zong, Z. NDM metallo- $\beta$-lactamases and their bacterial producers in health care settings. Clin. Microbiol. Rev. 2019, 32, e0115-18. [CrossRef]

34. Yang, F.; Mao, D.; Zhou, H.; Wang, X.; Luo, Y. Propagation of New Delhi Metallo- $\beta$-lactamase Genes (blaNDM-1) from a Wastewater Treatment Plant to Its Receiving River. Environ. Sci. Technol. Lett. 2016, 3, 138-143. [CrossRef]

35. Wang, R.N.; Zhang, Y.; Cao, Z.H.; Wang, X.Y.; Ma, B.; Wu, W.B.; Hu, N.; Huo, Z.Y.; Yuan, Q.B. Occurrence of super antibiotic resistance genes in the downstream of the Yangtze River in China: Prevalence and antibiotic resistance profiles. Sci. Total Environ. 2019, 651, 1946-1957. [CrossRef] [PubMed]

36. Yang, F.; Huang, L.; Li, L.; Yang, Y.; Mao, D.; Luo, Y. Discharge of KPC-2 genes from the WWTPs contributed to their enriched abundance in the receiving river. Sci. Total Environ. 2017, 581, 136-143. [CrossRef] [PubMed]

37. Xu, G.; Jiang, Y.; An, W.; Wang, H.; Zhang, X. Emergence of KPC-2-producing Escherichia coli isolates in an urban river in Harbin, China. World J. Microbiol. Biotechnol. 2015, 31, 1443-1450. [CrossRef] [PubMed]

38. Piotrowska, M.; Przygodzinska, D.; Matyjewicz, K.; Popowska, M. Occurrence and Variety of beta-Lactamase Genes among Aeromonas spp. Isolated from Urban Wastewater Treatment Plant. Front. Microbiol. 2017, 8, 863. [CrossRef] [PubMed] 
39. Harnisz, M.; Korzeniewska, E. The prevalence of multidrug-resistant Aeromonas spp. in the municipal wastewater system and their dissemination in the environment. Sci. Total Environ. 2018, 626, 377-383. [CrossRef] [PubMed]

40. Sekizuka, T.; Inamine, Y.; Segawa, T.; Hashino, M.; Yatsu, K.; Kuroda, M. Potential KPC-2 carbapenemase reservoir of environmental Aeromonas hydrophila and Aeromonas caviae isolates from the effluent of an urban wastewater treatment plant in Japan. Environ. Microbiol. Rep. 2019, 11, 589-597. [CrossRef]

41. Jang, H.M.; Kim, Y.B.; Choi, S.; Lee, Y.; Shin, S.G.; Unno, T.; Kim, Y.M. Prevalence of antibiotic resistance genes from effluent of coastal aquaculture, South Korea. Environ. Pollut. 2018, 233, 1049-1057. [CrossRef]

(C) 2020 by the authors. Licensee MDPI, Basel, Switzerland. This article is an open access article distributed under the terms and conditions of the Creative Commons Attribution (CC BY) license (http://creativecommons.org/licenses/by/4.0/). 